\title{
Identification of major depressive disorder disease-related genes and functional pathways based on system dynamic changes of network connectivity
}

\author{
Ruijie Geng ${ }^{1,2}$ and Xiao Huang ${ }^{1,2^{*}}$
}

\begin{abstract}
Background: Major depressive disorder (MDD) is a leading psychiatric disorder that involves complex abnormal biological functions and neural networks. This study aimed to compare the changes in the network connectivity of different brain tissues under different pathological conditions, analyzed the biological pathways and genes that are significantly related to disease progression, and further predicted the potential therapeutic drug targets.

Methods: Expression of differentially expressed genes (DEGs) were analyzed with postmortem cingulate cortex (ACC) and prefrontal cortex (PFC) mRNA expression profile datasets downloaded from the Gene Expression Omnibus (GEO) database, including 76 MDD patients and 76 healthy subjects in ACC and 63 MDD patients and 63 healthy subjects in PFC. The co-expression network construction was based on system network analysis. The function of the genes was annotated by Kyoto Encyclopedia of Genes and Genomes (KEGG) pathway analysis. Human Protein Reference Database (HPRD, http://www.hprd.org/) was used for gene interaction relationship mapping.

Results: We filtered 586 DEGs in ACC and 616 DEGs in PFC for further analysis. By constructing the co-expression network, we found that the gene connectivity was significantly reduced under disease conditions $(P=0.04$ in PFC and $P=1.227 \mathrm{e}-09$ in ACC). Crosstalk analysis showed that CD19, PTDSS2 and NDST2 were significantly differentially expressed in ACC and PFC of MDD patients. Among them, CD19 and PTDSS2 have been targeted by several drugs in the Drugbank database. KEGG pathway analysis demonstrated that the function of CD19 and PTDSS2 were enriched with the pathway of Glycerophospholipid metabolism and T cell receptor signaling pathway.
\end{abstract}

Conclusion: Co-expression network and tissue comparing analysis can identify signaling pathways and cross talk genes related to MDD, which may provide novel insight for understanding the molecular mechanisms of MDD.

Keywords: Major depressive disorder, Anterior cingulate cortex, Prefrontal cortex, Co-expression network

\section{Background}

Major depressive disorder (MDD) is a leading psychiatric disorder, typically manifested as persistent depression, anhedonia, and occasional suicidal ideation and behavior

\footnotetext{
*Correspondence: huang.xiao@zs-hospital.sh.cn

1 Department of Psychological Medicine, Zhongshan Hospital, Fudan University, Shanghai 200032, China

Full list of author information is available at the end of the article
}

[1]. The 12-month prevalence of this psychotic mood disorder is $10.4 \%$, and the lifetime prevalence is $20.6 \%$ in United State [2]. MDD exerts negative effects on the quality of life and is also one of the leading causes of disability worldwide [3].

Although antidepressants are widely used at present, there are some limitations including long time treatment response (commonly weeks to months) and low response rates (one to two thirds will not respond 
to the first drug prescribed, and remain one third will not respond after multiple trials) [4-7]. The neuropathology mechanism underlying MDD remains unclear, which makes the diagnosis and treatment of depression be challenging.

In recent years, accumulating evidence suggests that depression is not only caused by a single brain region or a single gene abnormality but a disease with complex genetic characteristics and multiple etiologies. Widespread brain areas associated with "emotional network" were found to be abnormal in structure, function, and coordinated activity in MDD. Thus MDD is also considered as "disconnection syndrome". Disturbances in brain activity and impaired mood regulation are considered to be the main neuropathology underlying depression [8]. Beyond the hippocampus, anterior cingulate cortex (ACC) and prefrontal cortex (PFC) are also common abnormal areas in MDD [9]. ACC is involved in the modulation of negative affect, pain and cognitive control [10]. PFC plays an important role in the regulation of the hypothalamo-pituitary-adrenal (HPA)-axis in stress response and also depression. There is increasing evidence that MDD and chronic stress are associated with an excitatory inhibition (E: I) imbalance within PFC which is caused by a deficit of inhibitory synaptic transmission onto principal glutamatergic neurons [11]. A recent fMRI study showed that the functional connectivity of the medial PFC in MDD patients is reduced [12]. Accordingly, depression is a heterogeneous syndrome with distinct causes and pathophysiology.

Gene expression analysis has found a large number of genes and disease-related information in MDD. But due to heterogeneity and various sources of noise, the discovery of pathogenesis is still limited [13-15]. Considering the fact that complex phenotypes manifested in mammalian systems are the result of a complex array of networks operating within and between tissues, a network perspective is necessary to explain its etiology. Tissue-to-tissue network analysis provides a method for the identification of disease-specific genes in response to abnormalities of tissues based on genome-wide association studies [16, 17]. Converging evidence indicated that gene co-expression studies offer complementary perspectives on gene changes in the context of transcriptome studies [18]. Coexpression genes possibly shared similar functions, and they may arise via multiple and diverse biological pathways such as common regulatory pathways $[13,16,18]$. Dysfunction of signaling pathways is likely to induce a variety of pathologies [19]. Notably, by integrating multiple interactions among a large number of genes, the study of gene co-expression networks provides an approach to tackle the complexity of biological changes in polygenic disease $[13,20]$.
In this study, we systematically integrated the postmortem brain (ACC and PFC) datasets of MDD patients and healthy subjects and constructed DEGs co-expression networks. We hypothesize that genes with correlated expression patterns across tissues are more likely to be related to the disease. This will provide a novel and powerful framework to improve the understanding of the molecular mechanisms of MDD.

\section{Methods}

\section{Expression profile analysis}

Datasets of mRNA expression profiles were downloaded from GEO database, including six postmortem ACC datasets ('E-GEOD-54572', 'E-GEOD-54571', 'E-GEOD-54565', 'E-GEOD-54564', 'E-GEOD-54563', 'E-GEOD-54562') [21] and six PFC datasets ('E-GEOD-54570', 'E-GEOD-54568', 'E-GEOD-54567', 'E-GEOD-45642', 'E-GEOD-35978', 'E-GEOD-12654') [14, 21-23]. Gene ID was converted into a gene symbol through the platform transformation. Multiple probes may correspond to one gene, therefore these probes were combined using the 'WGCNA' package in R platform[24]. To merge the expression profile data of multiple batches and platforms under the same variance level, we performed Z-test correction on all expression profile data. After integrating the data, two expression profile datasets of the ACC and PFC were obtained. The 'limma' $\mathrm{R}$ package was used to identify the DEGs $(P<0.05, \mid \log 2$ (foldchange) $\mid>0$ ) [25].

\section{Correlation analysis and Co-expression network construction}

Compared with normal cellular homeostasis, gene expression pattern changes in disease conditions. The Changes in the correlation between genes can be used to identify critical genes related to the development of depression [26]. Therefore, we used the Pearson correlation coefficient with a threshold of 0.5 for correlation analysis to identify gene pairs that are significantly correlated between normal and disease states. Subsequently, we obtained gene co-expression relationships from the two brain tissues. The co-expression network was constructed by taken co-expression relationships as edges and genes as nodes. The isolated nodes and self-interactions were removed. Cytoscape software [27] (http:// www.cytoscape.org) was applied for the construction of the network.

\section{Comparative analysis on difference of network}

Generally speaking, gene interaction network follows power law distribution with stability and robustness. Compared with other genes, the hub genes in the network have a significantly higher number of connections. 
Gene connections in biological networks are dynamic and may lose or gain connections in the disturbed network under disease conditions [26]. Genes with altered connectivity or expression in disease are more likely to participate in disease progression and are expected to become therapeutic targets. Therefore, we statistically measured the gain or loss of nodes in the normal and disease networks.

\section{Functional pathway analysis}

To further understand the biological functions of the DEGs from the two brain tissues, functional enrichment analysis was performed using KEGG (the Kyoto Encyclopedia of Genes and Genomes) pathway enrichment analysis (http://www.kegg.jp/). Enrichment methods used Fisher's exact test, the $P$ values were adjusted by FDR (false discovery rate). Signaling pathways with $P<0.05$ were considered as significantly enriched pathways.

\section{Cross talk analysis}

Biological processes are interconnected and regulated by signal proteins. The pathways that influence the dynamics of each other are collectively called cross-talk [28]. Previous studies have found the possibility of crosstalk genes as important drug targets and biomarkers of diseases [29]. We used the DEGs and their enriched signaling pathways to construct a regulatory network. Human
Protein Reference Database (HPRD, http://www.hprd. org/) was used for gene interaction relationship mapping. Based on the distribution of significant DEGs in each pathway, we identified crucial cross-talk genes that have a function in multiple important biological pathways.

\section{Results}

\section{Expression profile analysis}

The integrated datasets of ACC contained 152 samples, including 76 disease samples and 76 healthy controls. While integrated datasets of PFC included 126 samples with 63 disease samples and 63 healthy controls. After differential gene analysis, 586 DEGs were obtained in ACC (Fig. 1a) and 616 DEGs in PFC (Fig. 1b). More than $50 \%$ of DEGs in the two tissues were dysregulated and most DEGs were down-regulated (Fig. 1c).

\section{Co-expression network construction}

We constructed co-expression networks based on the correlation between genes by Cytoscape software (Fig. 2) and analyzed the topological properties of these networks. Compared with normal conditions, there is no significant change in the number of nodes in the network under disease conditions, but the connectivity between genes is significantly reduced (Table 1). We used Wilcox test to calculate $p$-value for the difference in gene connectivity between normal and disease conditions. The

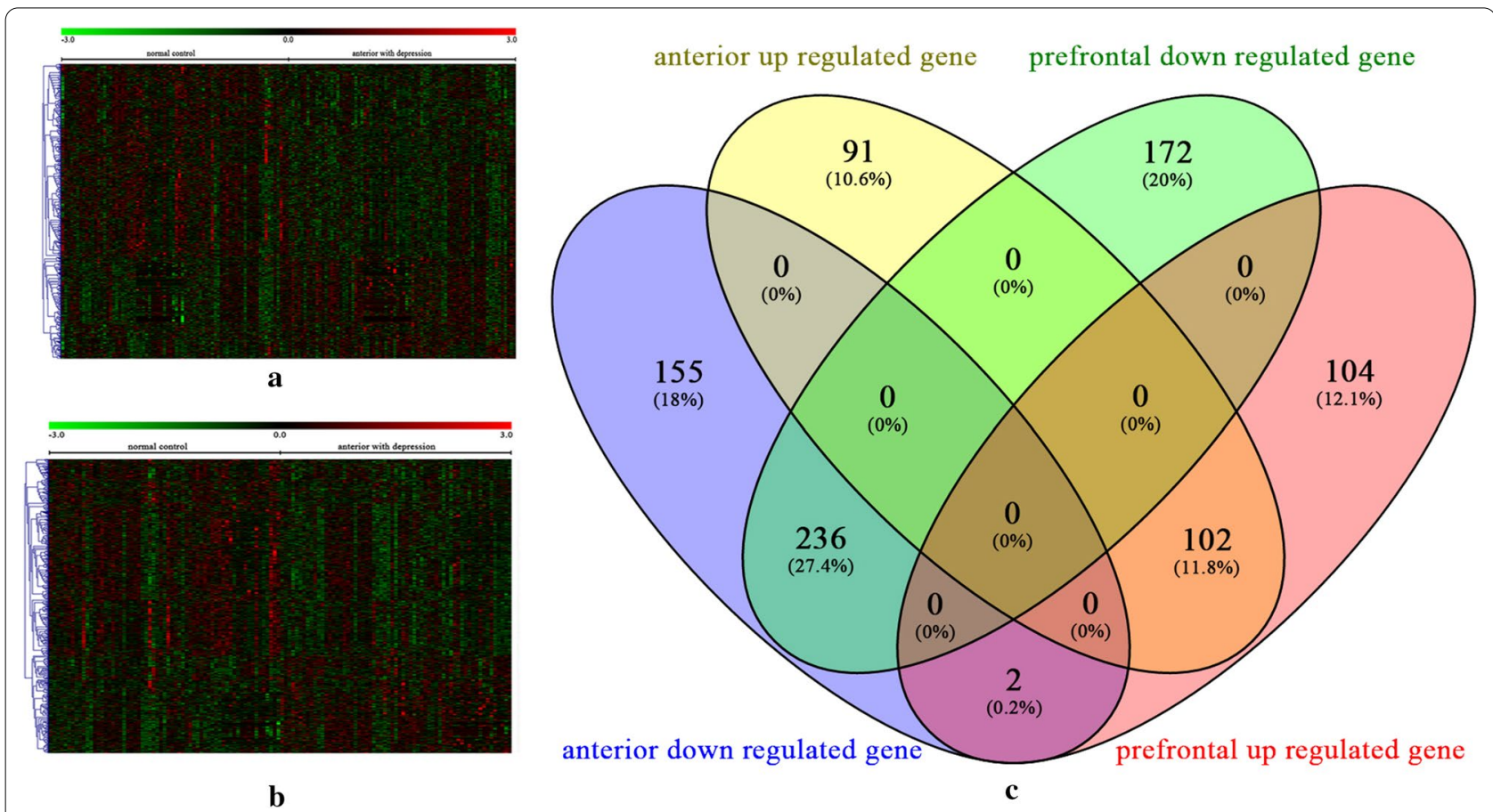

Fig. 1 The differentially expressed genes (DEGs) in ACC and PFC of patients with depression. $\mathbf{a}$, b Heatmap of DEGs in ACC and PFC, respectively. $\mathbf{c}$ The venn graph of DEGs between ACC and PFC. ACC, anterior cingulate cortex; PFC, prefrontal cortex 


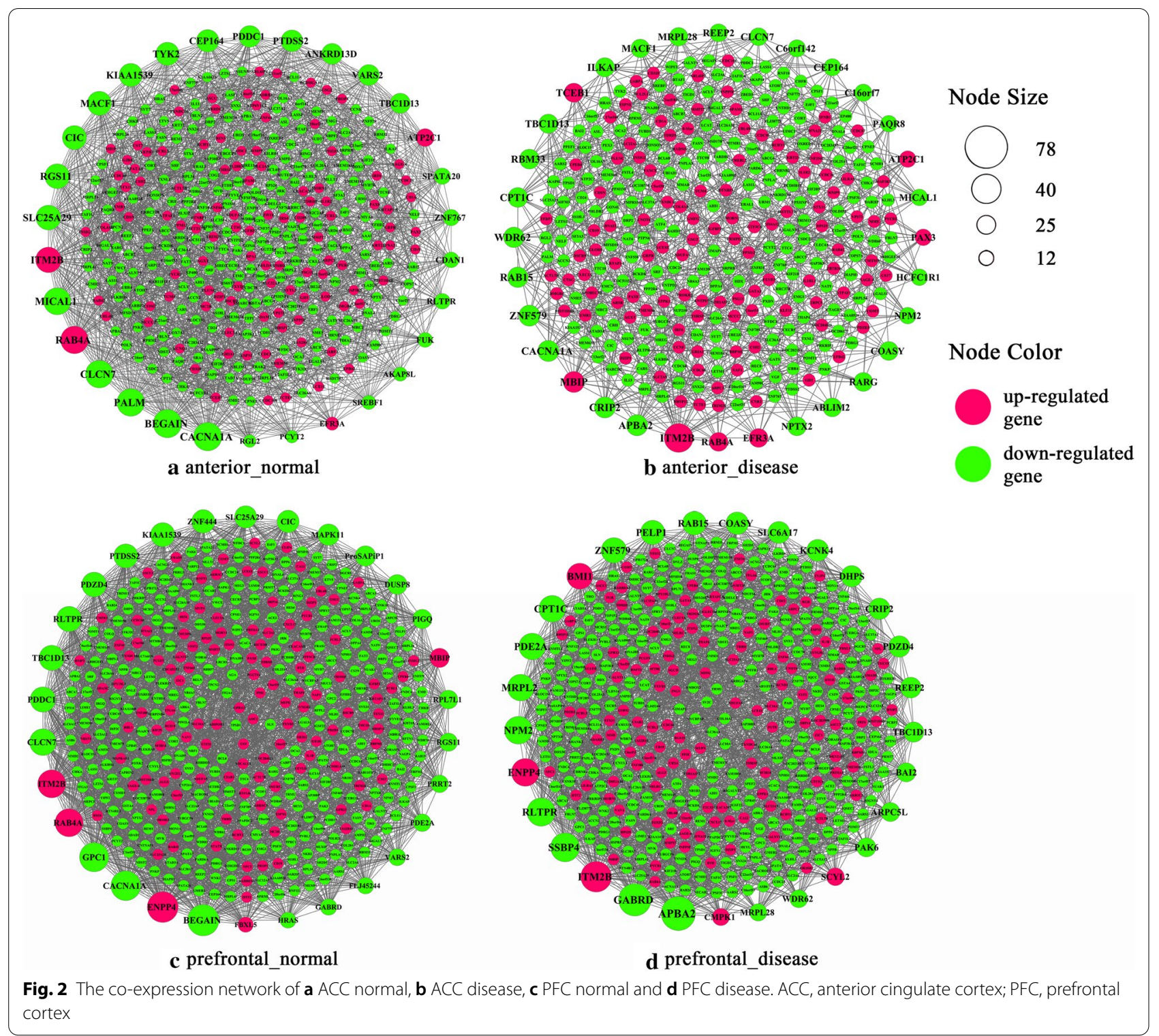

Table 1 The comparison of network topological properties

\begin{tabular}{lllll}
\hline summary & Anterior_normal & Anterior_disease & Prefrontal_normal & $\begin{array}{l}\text { Prefrontal_ } \\
\text { disease }\end{array}$ \\
\hline Nodes & 388 & 346 & 465 & 472 \\
Edges & 1604 & 735 & 2941 & 2287 \\
Unconnected nodes & 89 & 111 & 68 & 92 \\
Clustering coefficient & 0.295 & 0.181 & 0.301 & 0.275 \\
Density & 0.021 & 0.012 & 0.027 & 0.021 \\
centralization & 0.101 & 0.078 & 0.141 & 0.118 \\
\hline
\end{tabular}


results showed that $P=0.04$ in PFC and $P=1.227 \mathrm{e}-09$ in ACC. This suggested that under disease conditions, especially in ACC, there are statistically significant differences in the gain or loss of gene linkage.

\section{Comparative analysis on difference of networks}

Network topology analysis reveals that gene connectivity was decreased in MDD patients. To further reveal the correlation of the two tissue lesions and MDD, we compared the nodes with gain and loss of connections (Fig. 3a, b) in the two disease networks. The number of nodes with a gain of connections was nearly balanced with the loss in PFC (Fig. 3c). Our data also showed that the number of nodes with a gain of connections in the PFC network was higher than that in the ACC network with the ratio of 1.72:1, while the numbers of nodes with loss of connections tended to be similar in the two networks with the ratio of 1.02:1. The probability density distribution of the co-expression network showed that the variance of the density distribution in ACC increases significantly (Fig. 3d), while it tends to be normal in PFC (Fig. 3e).

\section{Functional pathway analysis}

To obtain information about the biological effects of DEGs, we performed the functional enrichment analysis on up-regulated and down-regulated DEGs. We found that DEGs in ACC were mainly associated with circulatory system related pathways (Fig. 4a), while DEGs in PFC were enriched in metabolic system related pathways (Fig. 4b). By comparing the gene count and the $P$ value of the pathway, it is found that the P-value of the pathway increases with the increase of gene count (Fig. 4c). We further performed cross talk analysis on these DEGs enriched pathways, to identify the cross talk genes that regulate multiple signaling pathways.

\section{Cross talk analysis}

Signaling pathways and genes enriched in pathways were used to establish a pathway-gene complex network including 219 relationship pairs, 16 signaling pathways and 70 genes (Fig. 5a). The signaling pathways and genes with the highest degrees ranking as top 10 were extracted (Table 2). These top10 pathways and genes are more likely to be involved in the development of MDD and may also be potential new therapeutic targets.
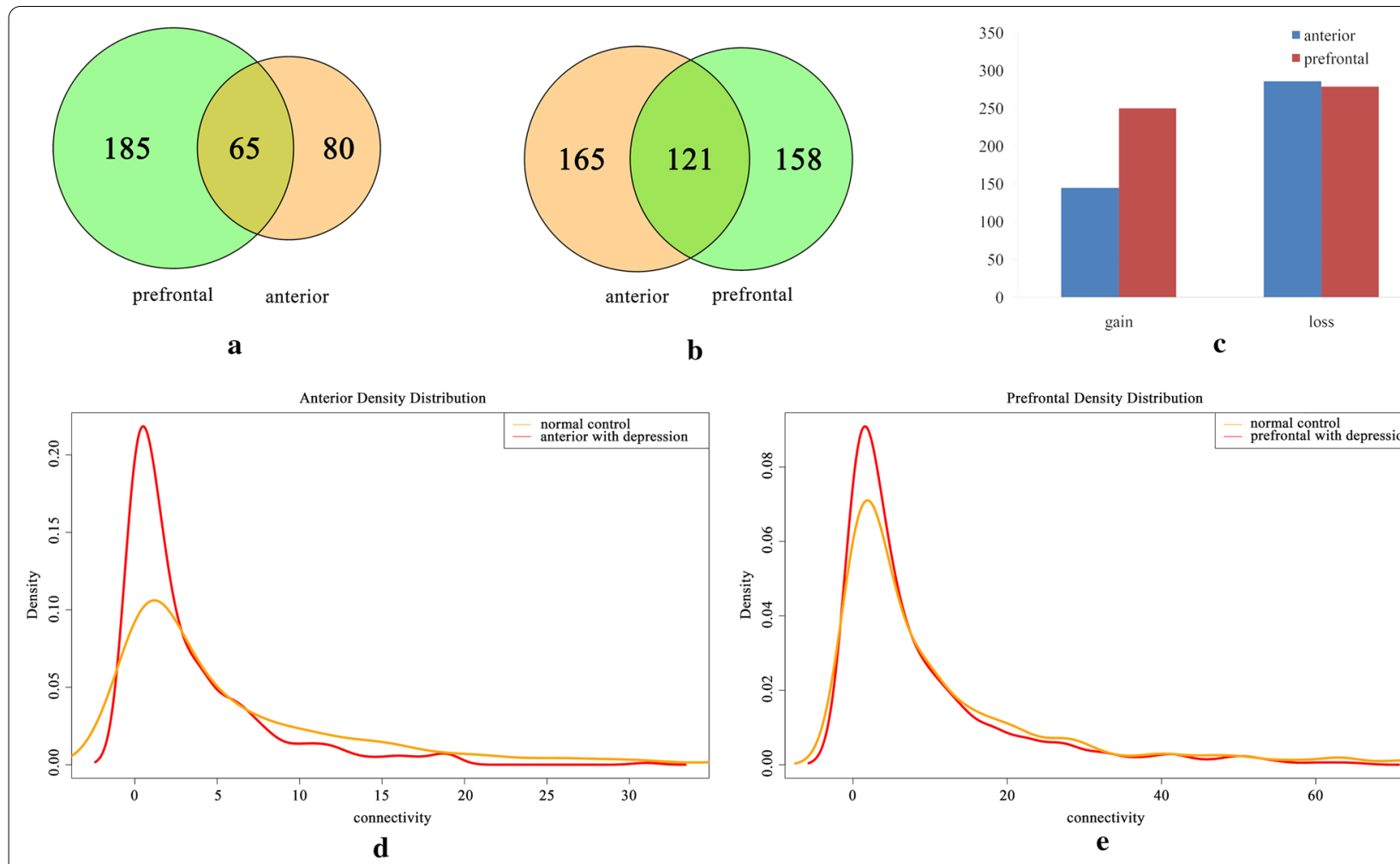

b

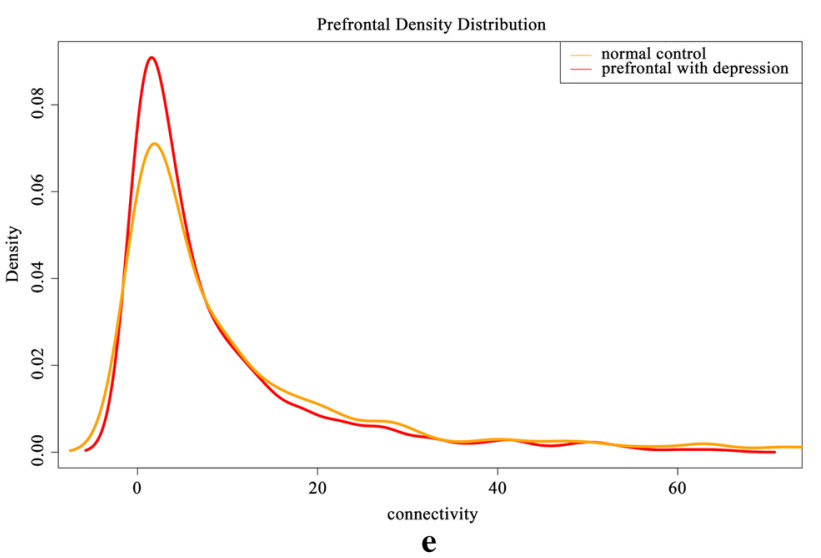

Fig. 3 Comparative analysis of network difference. a The node counts of gain of connections between ACC and PFC; $\mathbf{b}$ The node counts of loss of connections between ACC and PFC; c Comparison of nodes with gain or loss of connections in ACC and PFC; $\mathbf{d}$ Probability density distribution of co-expression network in ACC under normal and depression conditions; e probability density distribution of co-expression network in PFC under normal and depression conditions. ACC, anterior cingulate cortex; PFC, prefrontal cortex 


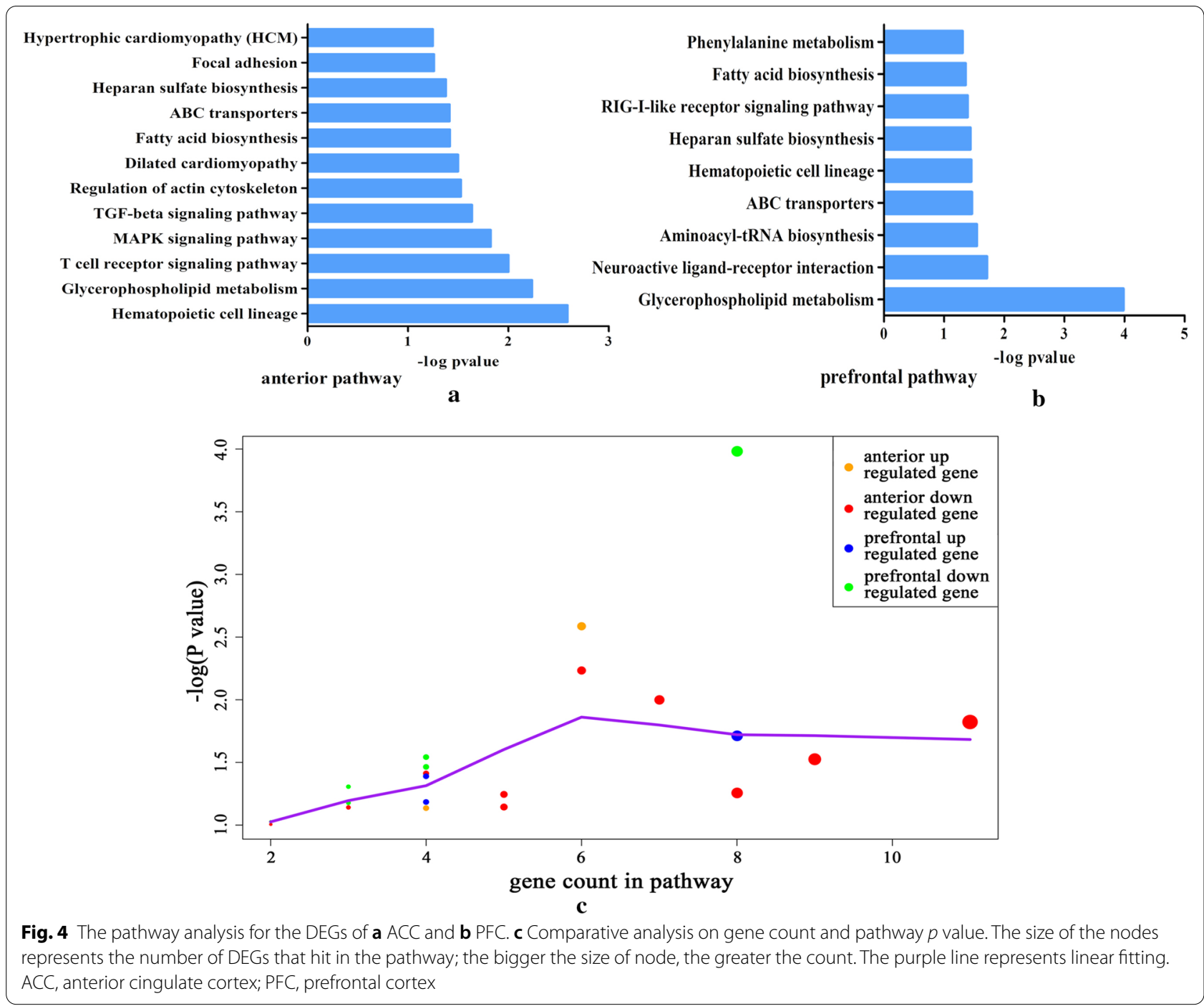

We further statistically analyzed the significance of the top10 genes through group comparison between ACC and PFC in MDD patients and healthy subjects (Table 3). Five genes (CACNA1A, PTDSS2, DIAPH1, CD19 and NDST2) in PFC and three genes (PTDSS2, CD19 and NDST2) in ACC were found differentially expressed in MDD patients (Table 3). Receiver operating characteristic (ROC) values of genes were calculated in the two tissues. All the ROC values of genes were higher than random state (0.5) (Fig. 5b and Table 3). To examine whether these top10 genes are capable to be potential novel therapeutic targets, we searched these genes in the Drugbank database. As shown in Table 4, five genes (CACNA1A, PTDSS2, $M A P K 11, C D 19$ and PAK6) were known to be targeted by several drugs. Except for Blinatumomab and KC706, other drugs have been reported to be related to brain tissue injury and cerebral nervous system diseases.

\section{Discussion}

In the current study, we analyzed DEGs in ACC and PFC from patients with MDD. Correlation networks based on co-expression were constructed. Topological properties of the networks were analyzed and compared. Our results showed that the lesions of brain tissues in MDD patients were not synchronized and alterations of biological functions were not consistent either. ACC showed a greater degree of abnormality as compared to PFC suggesting a higher correlation with disease progression. We consequently analyzed the signaling pathways enriched by DEGs and further cross talk genes that bridge the multiple pathways were also identified. Through the construction of the pathway-gene complex network, the genes and singling pathways with top10 degrees were extracted, which are more likely to be potential novel therapeutic targets. We also mined the drugbank database for the top10 cross talk genes to explore their drugable target 

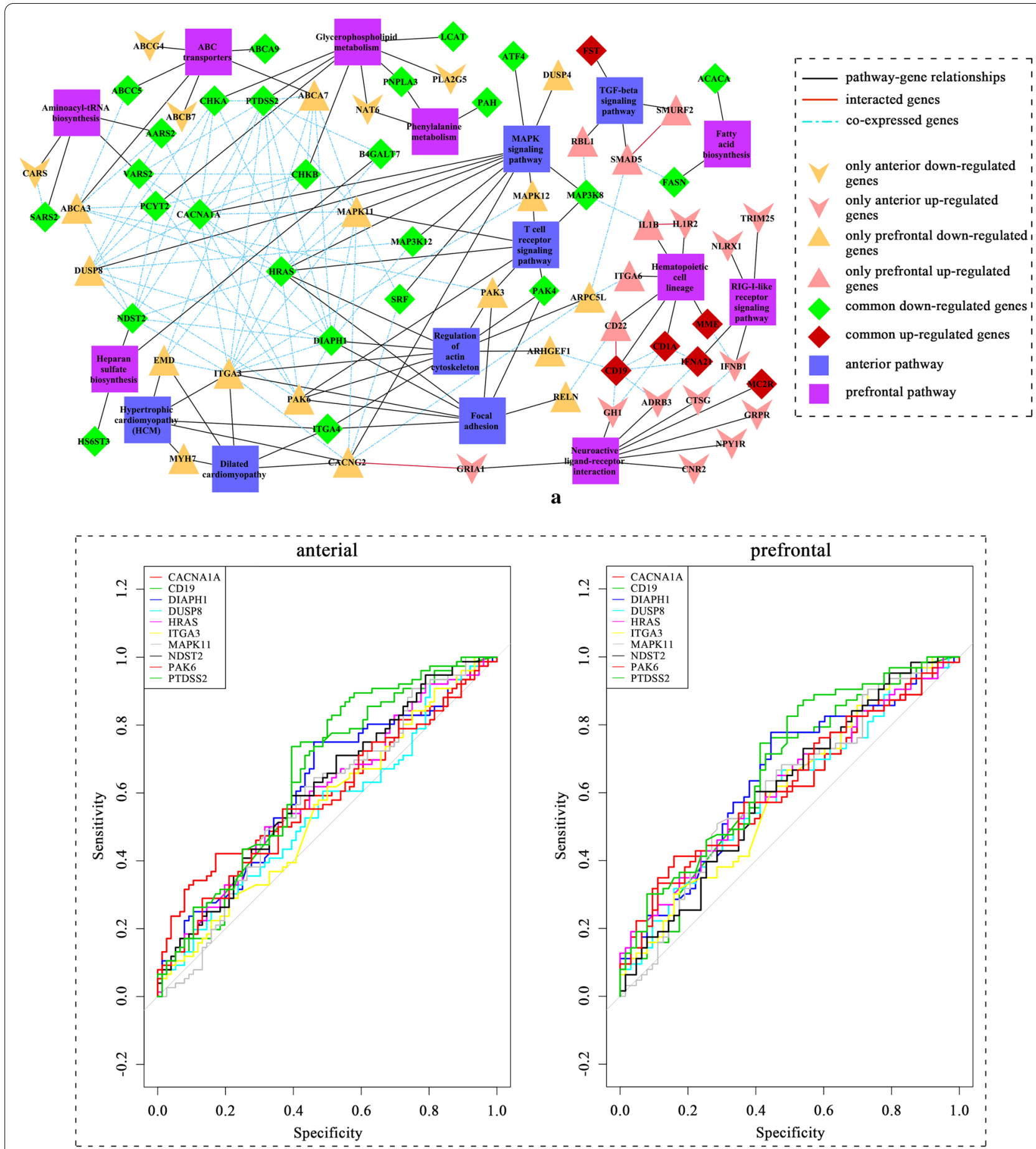

b

Fig. 5 The pathway-gene complex network. a Pathway-gene complex network. b The ROC curve of top 10 genes in network

potential. PTDSS2 and CD19 differentially expressed in both ACC and PFC may correlate with MDD progression, and are more likely to become the new drug targets for the treatment of MDD.
The co-expression network has the ability to mine functionally related genes with similar co-expression patterns [30], which have been widely used to identify candidate biomarkers and therapeutic targets for many 
Table 2 Top10 pathways and genes in the pathway-gene complex network

\begin{tabular}{llll}
\hline Pathway & Degree_path & Gene & Degree_gene \\
\hline Glycerophospholipid metabolism & 14 & CACNA1A & 22 \\
MAPK signaling pathway & 11 & PTDSS2 & 19 \\
Hematopoietic cell lineage & 10 & DIAPH1 & 15 \\
Regulation of actin cytoskeleton & 9 & ITGA3 & 14 \\
Focal adhesion & 8 & HRAS & 14 \\
Neuroactive ligand-receptor interaction & 8 & MAPK11 & 11 \\
ABC transporters & 8 & DUSP8 & 11 \\
T cell receptor signaling pathway & 7 & CD19 & 11 \\
Heparan sulfate biosynthesis & 6 & PAK6 & 10 \\
Dilated cardiomyopathy & 5 & NDST2 & 10 \\
\hline
\end{tabular}

Table 3 Significant analysis using top 10 genes

\begin{tabular}{lllllll}
\hline Gene & $\begin{array}{l}\boldsymbol{P} \text { value_anterior } \\
\text { vs control }\end{array}$ & $\begin{array}{l}\boldsymbol{p}_{\text {corrected anterior vs }} \\
\text { prefrontal }\end{array}$ & $\begin{array}{l}\boldsymbol{P} \text { value_prefrontal } \\
\text { vs control }\end{array}$ & $\begin{array}{l}\boldsymbol{p}_{\text {corrected }} \text { prefrontal } \\
\text { vs control }\end{array}$ & ROC_anterior & ROC_prefrontal \\
\hline CACNA1A & 0.031 & 0.062 & 0.031 & 0.044 & 0.606 & 0.615 \\
PTDSS2 & 0.003 & 0.013 & 0.009 & 0.030 & 0.641 & 0.643 \\
DIAPH1 & 0.016 & 0.054 & 0.008 & 0.039 & 0.616 & 0.644 \\
ITGA3 & 0.270 & 0.270 & 0.072 & 0.270 & 0.550 & 0.600 \\
HRAS & 0.032 & 0.054 & 0.022 & 0.054 & 0.598 & 0.620 \\
MAPK11 & 0.256 & 0.285 & 0.046 & 0.051 & 0.549 & 0.600 \\
DUSP8 & 0.086 & 0.122 & 0.030 & 0.050 & 0.583 & 0.610 \\
CD19 & 0.017 & 0.044 & 0.035 & 0.044 & 0.612 & 0.608 \\
PAK6 & 0.128 & 0.160 & 0.030 & 0.060 & 0.576 & 0.619 \\
NDST2 & 0.0007 & 0.008 & 0.0009 & 0.009 & 0.663 & 0.680 \\
\hline
\end{tabular}

Table 4 Drugable target information for the top 10 genes

\begin{tabular}{|c|c|c|c|c|}
\hline Gene & Target (yes/no) & Drug counts & Drugs & PMID \\
\hline CACNAIA & Yes & 4 & $\begin{array}{l}\text { Amlodipine, loperamide, lyrica, } \\
\text { pregabalin }\end{array}$ & $\begin{array}{r}25918454 \\
26390138 \\
26138193 \\
26670374\end{array}$ \\
\hline CD19 & Yes & 1 & Blinatumomab & \\
\hline PAK6 & Yes & 4 & $\begin{array}{l}\text { Dextromethorphan, Tizanidine, } \\
\text { Agmatine, Moxonidine }\end{array}$ & $\begin{array}{r}26471212 \\
23648652 \\
26678503 \\
24333661\end{array}$ \\
\hline MAPK11 & Yes & 2 & KC706, Regorafenib & 25563977 \\
\hline PTDSS2 & Yes & 1 & Phosphatidylserine & 26689775 \\
\hline DIAPH1 & No & 0 & & \\
\hline ITGA3 & No & 0 & & \\
\hline HRAS & No & 0 & & \\
\hline DUSP8 & No & 0 & & \\
\hline NDST2 & No & 0 & & \\
\hline
\end{tabular}


complex diseases, such as Alzheimer's disease, schizophrenia and cancer [31-33]. Besides, the network perspective supports the high heterogeneity of depression and explains how different treatment methods might take effect $[8,20]$. Comparisons between many data sets can provide a global view of gene expression patterns across tissues [16]. Therefore, we completed a comprehensive analysis of gene expressions across ACC and PFC in patients with MDD and healthy subjects. Through analyzing and comparing the four coexpression networks, we found the unconnected nodes were increased in disease condition, which may be due to loss of connections. Alteration in important nodes of the network may affect the function of the entire network, causing depression. The topology analysis of the co-expression network showed that in disease states, the number of nodes with a gain of connections in PFC network higher than that in ACC network with the ratio of 1.72:1 and variance of density distribution was markedly increased in the ACC network, but there is no significant change in PFC. These results indicated that the PFC network status of patients with depression tended to be normal, while the ACC network presented drastic fluctuations. The stability of the PFC network and its resistance to disease signals are better than that of the ACC network, which was consistent with the results of the Wilcox test. It revealed that the pathological changes of brain tissues in depression patients were not synchronized and alterations of biological functions were not consistent either. Compared with PFC, ACC showed a higher degree of abnormality and may have a strong correlation with disease progression. Therefore, ACC is more likely to be a therapeutic target for depression. ACC is located in the frontal part of the cingulate cortex inside the cerebral hemispheres and is a part of the limbic system. Substantial evidence from healthy subjects has linked the ACC to emotional behavior [10, 34]. This brain area uses information about punishment to manage aversively motivated actions. Bush et al. compiled a large amount of functional imaging, electrophysiological and anatomical data, and found that the ACC is specialized for affective processes [35]. Philippi et al. used resting-state fMRI to examine the functional connectivity of the ACC subregion in 28 participants with subclinical levels of depression. The results suggested that there is a clear correlation between depression severity and functional connectivity of ACC subregions. The reduced pregenual ACC-striatum connectivity and anterior subgenual ACC -anterior insula connectivity was related to higher depression severity [36]. Similarly, our research also found that ACC network connectivity in patients with depression has decreased, which is consistent with previous studies.

Signaling molecules commonly do not work individually but interact with other proteins or biological molecules to achieve signal transmission. for gaining further understanding of MDD. Moreover, these signaling components which may co-expressed in a dataset and correlate across samples are predicted to reconstruct multiple signaling pathways and their cross-talk maps for further biomedical research [16]. Cross-talk analysis is commonly used to explore the regulation and cooperation between signaling pathways, and further reveal the pathogenesis of diseases [37]. Therefore, through analyzing the pathway-gene network, we identified ten pathways and ten cross talk genes with highest degrees. Among these 10 genes, CD19, PTDSS2 and NDST2 were significantly differentially expressed in ACC and PFC of MDD patients. Moreover, CD19 and PTDSS2 have been targeted by several drugs. Therefore, these two genes may be related to the progression of MDD or other neurological diseases, and are more likely to become the new drug targets for the treatment of MDD.

Phosphatidylserine synthase 2 (PTDSS2) can convert phosphatidylethanolamine (PE) into phosphatidylserine(PS) and participate in important cell signaling processes $[38,39]$. Compared with other tissues, brain is enriched in PS and PE. Besides, $>36 \%$ of the PS are composed of docosahexaenoic acid (DHA) which is essential for normal function of the nervous system $[40,41]$. Studies have shown that the reduction of DHA is associated with the development of mild cognitive impairment to Alzheimer's disease [42]. Similarly, we observed that the most significant pathway is Glycerophospholipid metabolism. Both this pathway and PTDSS2 are related to lipid metabolism. Recent studies have shown that meningeal lipids play an important role in the pathogenesis of depressive disorder and anxiety [39]. The typical glycerophospholipids (GPLs) found in mammalian membranes include phosphatidylcholines (PC), PE, PS and phosphatidylinositols (PI) that are all attached through a phosphodiester linkage [39]. Preclinical findings indicated that the membrane-forming n-3 polyunsaturated fatty acids, glycerolipids, GPLs, and sphingolipids (SPLs) play a crucial role in the induction of depression- and anxiety-related behaviors [43]. Clinical studies suggested that compared with non-depressed non-suicide subjects, the activities of phosphatidylinositol 3-kinase (PI3K) and Akt (serine threonine kinase or protein kinase $B$ ) in MDD patients were significantly reduced [44]. Another crucial gene, CD19, is a B cellspecific member of the immunoglobulin superfamily expressed by pre- $B$ cells from the time of heavy chain rearrangement to final differentiation into plasma cells. 
By regulating $\mathrm{B}$ cell receptor signaling, $C D 19$ guides the fate of B cells and differentiation lymphopoiesis [45]. In our study, CD19 is the top gene in the $\mathrm{T}$ cell receptor signaling pathway that participates in immune regulation and inflammatory response. Immunity dysfunction is a risk factor for depression. Large clinical cohort studies have found that autoimmune diseases or severe infections increase the risk of mood disorders [46]. The activation of innate immune cells produces pro-inflammatory cytokines, which can cause major depressive disorder by inhibiting monoamine neurotransmitters, activating the HPA axis, and affecting neurogenesis and plasticity [47]. From the clinical perspective, anti-inflammatory drugs, such as minocycline, have been reported to cause improvement in patients with treatment-resistant depression [48]. Taken together, improving lipid metabolism and regulating inflammatory response can provide new directions for the prevention and treatment of MDD.

Our research has identified several critical genes and provided some interesting clues for further experiments. However, some limitations of the study should be mentioned. First, we identified several genes from microarray data analysis. But we did not perform further functional verification of these selected genes. Subsequently, a large number of clinical samples will be needed to validate our findings and clarify the underlying mechanisms of how these genes affect the pathological stage. Another limitation of the study is the AUC of the curve is low, although the $\mathrm{ROC}$ values of all genes are higher than the random state (0.5). Therefore, the interpretation of this result needs to be cautious. Further exploration is needed in the future.

\section{Conclusions}

In conclusion, this study can identify several crucial genes for future genetic association studies. It also proved the essence of integrating cross-tissue data, gene co-expression and crosstalk signaling results, paving the way for novel and complementary approaches to investigate the molecular pathology of MDD and other complex brain disorders.

\footnotetext{
Abbreviations

MDD: Major depressive disorder; ACC: Postmortem anterior cingulate cortex; PFC: Prefrontal cortex; DEGs: Differentially expressed genes; KEGG: The Kyoto Encyclopedia of Genes and Genomes); FDR: False discovery rate; HPRD: Human Protein Reference Database; ROC: Receiver operating characteristic: BDNF: Brain-derived neurotrophic factor; GABA: Gamma amino acid butyric acid; GPLs: Glycerophospholipids; PC: Phosphatidylcholines; PE: Phosphatidylethanolamines; PS: Phosphatidylserines; PI: Phosphatidylinositols; PI3K: Phosphatidylinositol-3 kinase; SPLs: Sphingolipids; mTORC1: MTOR complex 1; PSD95: Postsynaptic density-95; PTDSS2: Phosphatidylserine synthase 2; PAKs: P21-activated kinases; AR: Androgen receptor; GR: Glucocorticoid receptor.
}

Acknowledgements

We thank anonymous reviewers for their useful comments on the manuscript.

\section{Authors' contributions}

$\mathrm{XH}$ designed the study, analyzed the data, performed computational coding. XH and RG involved in drafting the manuscript and revising it critically for important intellectual content. All authors read and approved the final manuscript.

\section{Funding}

The study design and data collections were supported by the Construction of Public Health System in Shanghai (Mental and Mental Health) (No. GWV-10.2-XD26). The analysis and interpretation of data, and the writing of the manuscript were supported by the Education Foundation of Zhongshan Hospital, Fudan University (No. 051).

\section{Availability of data and materials}

The GeneChip data were retrieved from the GEO data repository (http://www. ncbi.nlm.nih.gov/geo/) with the accession numbers GSE54572, GSE54571, GSE54565, GSE54564, GSE54563, GSE54562, GSE54570, GSE54568, GSE54567, GSE45642, GSE35978, GSE12654.

Ethics approval and consent to participate

Not applicable.

Consent for publication

Not applicable.

\section{Competing interests}

The authors declare that they have no competing interests.

\begin{abstract}
Author details
${ }^{1}$ Department of Psychological Medicine, Zhongshan Hospital, Fudan University, Shanghai 200032, China. ${ }^{2}$ Department of Psychological Medicine, Xiamen Branch, Zhongshan Hospital, Fudan University, Xiamen 361015, China.
\end{abstract}

Received: 21 October 2019 Accepted: 17 February 2021

Published online: 23 February 2021

\section{References}

1. Otte C, Gold SM, Penninx BW, Pariante CM, Etkin A, Fava M, et al. Major depressive disorder. Nat Rev Dis Primers. 2016;2:16065.

2. Hasin DS, Sarvet AL, Meyers JL, Saha TD, Ruan WJ, Stohl M, et al. Epidemiology of adult DSM-5 major depressive disorder and its specifiers in the United States. JAMA Psychiatry. 2018;75(4):336-46.

3. Inserra A, Mastronardi CA, Rogers G, Licinio J, Wong ML. Neuroimmunomodulation in major depressive disorder: focus on caspase 1 , inducible nitric oxide synthase, and interferon-gamma. Mol Neurobiol. 2019;56(6):4288-305.

4. Berlim MT, Fleck MP, Turecki G. Current trends in the assessment and somatic treatment of resistant/refractory major depression: an overview. Ann Med. 2008;40(2):149-59.

5. Cain RA. Navigating the Sequenced Treatment Alternatives to Relieve Depression (STAR*D) study: practical outcomes and implications for depression treatment in primary care. Prim Care. 2007:34(3):505-19, vi.

6. Little A. Treatment-resistant depression. Am Fam Physician. 2009:80(2):167-72.

7. Huang SH, LePendu P, Iyer SV, Tai-Seale M, Carrell D, Shah NH. Toward personalizing treatment for depression: predicting diagnosis and severity. J Am Med Inform Assoc: JAMIA. 2014;21(6):1069-75.

8. Song Z, Zhang M, Huang P. Aberrant emotion networks in early major depressive disorder patients: an eigenvector centrality mapping study. Transl Psychiatry. 2016;6:e819.

9. Rodriguez-Cano E, Sarro S, Monte GC, Maristany T, Salvador R, McKenna PJ, et al. Evidence for structural and functional abnormality in the subgenual anterior cingulate cortex in major depressive disorder. Psychol Med. 2014;44(15):3263-73. 
10. Tolomeo S, Christmas D, Jentzsch I, Johnston B, Sprengelmeyer R, Matthews $\mathrm{K}$, et al. A causal role for the anterior mid-cingulate cortex in negative affect and cognitive control. Brain. 2016;139(Pt 6):1844-54.

11. Fogaca MV, Duman RS. Cortical GABAergic dysfunction in stress and depression: new insights for therapeutic interventions. Front Cell Neurosci. 2019;13:87.

12. Murrough JW, Abdallah CG, Anticevic A, Collins KA, Geha P, Averill LA, et al. Reduced global functional connectivity of the medial prefrontal cortex in major depressive disorder. Hum Brain Mapp. 2016.

13. Gaiteri C, Guilloux JP, Lewis DA, Sibille E. Altered gene synchrony suggests a combined hormone-mediated dysregulated state in major depression. PLoS ONE. 2010;5(4):e9970.

14. Li JZ, Bunney BG, Meng F, Hagenauer MH, Walsh DM, Vawter MP, et al. Circadian patterns of gene expression in the human brain and disruption in major depressive disorder. Proc Natl Acad Sci USA. 2013;1 10(24):9950-5.

15. Wang Q, Dwivedi Y. Transcriptional profiling of mitochondria associated genes in prefrontal cortex of subjects with major depressive disorder. World J Biol Psychiatry. 2016:1-29.

16. Dobrin R, Zhu J, Molony C, Argman C, Parrish ML, Carlson S, et al. Multitissue coexpression networks reveal unexpected subnetworks associated with disease. Genome Biol. 2009;10(5):1-13.

17. Geng R, Li Z, Yu S, Yuan C, Hong W, Wang Z, et al. Weighted gene coexpression network analysis identifies specific modules and hub genes related to subsyndromal symptomatic depression. The world journal of biological psychiatry : the official journal of the World Federation of Societies of Biological Psychiatry. 2018:1-23.

18. Lee HK, Hsu AK, Sajdak J, Qin J, Pavlidis P. Coexpression analysis of human genes across many microarray data sets. Genome Res. 2004;14(6):1085-94.

19. Hughey JJ, Lee TK, Covert MW. Computational modeling of mammalian signaling networks. Wiley Interdiscip Rev Syst Biol Med. 2010;2(2):194-209.

20. Gaiteri C, Ding Y, French B, Tseng GC, Sibille E. Beyond Modules \& Hubs: the potential of gene coexpression networks for investigating molecular mechanisms of complex brain disorders. Genes Brain Behav. 2014;13(1):13-24.

21. Chang L-C, Jamain S, Lin C-W, Rujescu D, Tseng GC, Sibille E. A conserved BDNF, glutamate- and GABA-enriched gene module related to human depression identified by coexpression meta-analysis and DNA variant genome-wide association studies. PLoS ONE. 2014;9(3):e90980.

22. Chen C, Cheng L, Grennan K, Pibiri F, Zhang C, Badner JA, et al. Two gene co-expression modules differentiate psychotics and controls. Mol Psychiatry. 2013;18(12):1308-14.

23. I wamoto K, Kakiuchi C, Bundo M, Ikeda K, Kato T. Molecular characterization of bipolar disorder by comparing gene expression profiles of postmortem brains of major mental disorders. Mol Psychiatry. 2004;9(4):406-16

24. Langfelder P, Horvath S. WGCNA: an R package for weighted correlation network analysis. BMC Bioinformatics. 2008;9:559.

25. Diboun I, Wernisch L, Orengo CA, Koltzenburg M. Microarray analysis after RNA amplification can detect pronounced differences in gene expression using limma. BMC Genomics. 2006;7:252.

26. Kaushik A, Bhatia Y, Ali S, Gupta D. Gene network rewiring to study melanoma stage progression and elements essential for driving melanoma. PLoS ONE. 2015;10(11):e0142443.

27. Lopes CT, Franz M, Kazi F, Donaldson SL, Morris Q, Bader GD. Cytoscape web: an interactive web-based network browser. Bioinformatics. 2010:26(18):2347-8.

28. Zhao CY, Greenstein JL, Winslow RL. Roles of phosphodiesterases in the regulation of the cardiac cyclic nucleotide cross-talk signaling network. J Mol Cell Cardiol. 2016;91:215-27.

29. Mei S, Zhu H. Multi-label multi-instance transfer learning for simultaneous reconstruction and cross-talk modeling of multiple human signaling pathways. BMC Bioinform. 2015;16:417.
30. van Dam S, Võsa U, van der Graaf A, Franke L, de Magalhães JP. Gene co-expression analysis for functional classification and gene-disease predictions. Brief Bioinform. 2018;19(4):575-92.

31. Kakati T, Bhattacharyya DK, Barah P, Kalita JK. Comparison of methods for differential co-expression analysis for disease biomarker prediction. Comput Biol Med. 2019;1 13:103380.

32. Zhu L, Ding Y, Chen CY, Wang L, Huo Z, Kim S, et al. MetaDCN: metaanalysis framework for differential co-expression network detection with an application in breast cancer. Bioinformatics (Oxford, England). 2017;33(8):1121-9.

33. Fromer M, Roussos P, Sieberts SK, Johnson JS, Kavanagh DH, Perumal TM, et al. Gene expression elucidates functional impact of polygenic risk for schizophrenia. Nat Neurosci. 2016;19(11):1442-53.

34. Ganesh G, Minamoto T, Haruno M. Activity in the dorsal ACC causes deterioration of sequential motor performance due to anxiety. Nat Commun. 2019;10(1):4287.

35. Bush G, Luu P, Posner MI. Cognitive and emotional influences in anterior cingulate cortex. Trends Cognit Sci. 2000;4(6):215-22.

36. Philippi CL, Motzkin JC, Pujara MS, Koenigs M. Subclinical depression severity is associated with distinct patterns of functional connectivity for subregions of anterior cingulate cortex. J Psychiatr Res. 2015;71:103-11.

37. Kassiri Z, Defamie V, Hariri M, Oudit GY, Anthwal S, Dawood F, et al. Simultaneous transforming growth factor beta-tumor necrosis factor activation and cross-talk cause aberrant remodeling response and myocardial fibrosis in Timp3-deficient heart. J Biol Chem. 2009;284(43):29893-904.

38. Kimura AK, Kim HY. Phosphatidylserine synthase 2: high efficiency for synthesizing phosphatidylserine containing docosahexaenoic acid. J Lipid Res. 2013;54(1):214-22.

39. Muller CP, Reichel M, Muhle C, Rhein C, Gulbins E, Kornhuber J. Brain membrane lipids in major depression and anxiety disorders. Biochim Biophys Acta. 2015;1851(8):1052-65.

40. Kim HY, Huang BX, Spector AA. Phosphatidylserine in the brain: metabolism and function. Prog Lipid Res. 2014;56:1-18.

41. Glade MJ, Smith K. Phosphatidylserine and the human brain. Nutrition. 2015:31(6):781-6.

42. Cunnane SC, Schneider JA, Tangney C, Tremblay-Mercier J, Fortier M, Bennett DA, et al. Plasma and brain fatty acid profiles in mild cognitive impairment and Alzheimer's disease. J Alzheimers Dis. 2012;29(3):691-7.

43. Reichel M, Honig S, Liebisch G, Luth A, Kleuser B, Gulbins E, et al. Alterations of plasma glycerophospholipid and sphingolipid species in male alcohol-dependent patients. Biochim Biophys Acta. 2015;1851(11):1501-10

44. Karege F, Perroud N, Burkhardt S, Fernandez R, Ballmann E, La Harpe R, et al. Alterations in phosphatidylinositol 3-kinase activity and PTEN phosphatase in the prefrontal cortex of depressed suicide victims. Neuropsychobiology. 2011;63(4):224-31.

45. Watkins MP, Bartlett NL. CD19-targeted immunotherapies for treatment of patients with non-Hodgkin B-cell lymphomas. Expert Opin Investig Drugs. 2018;27(7):601-11.

46. Benros ME, Waltoft BL, Nordentoft M, Ostergaard SD, Eaton WW, Krogh J, et al. Autoimmune diseases and severe infections as risk factors for mood disorders: a nationwide study. JAMA Psychiatry. 2013;70(8):812-20.

47. Dantzer R, O'Connor JC, Freund GG, Johnson RW, Kelley KW. From inflammation to sickness and depression: when the immune system subjugates the brain. Nat Rev Neurosci. 2008;9(1):46-56.

48. Husain MI, Chaudhry IB, Husain N, Khoso AB, Rahman RR, Hamirani MM, et al. Minocycline as an adjunct for treatment-resistant depressive symptoms: A pilot randomised placebo-controlled trial. J Psychopharmacol. 2017;31(9):1166-75.

\section{Publisher's Note}

Springer Nature remains neutral with regard to jurisdictional claims in published maps and institutional affiliations. 